\title{
CONOCIMIENTOS, ACTITUDES Y PRÁCTICAS DE ADOLESCENTES ESPAÑOLES SOBRE LA VIOLENCIA DE PAREJA
}

\section{KNOWLEDGE, ATTITUDES AND PRACTICES OF SPANISH ADOLESCENTS ON INTIMATE PARTNER VIOLENCE}

\author{
María del Mar Pastor Bravo. University of Toronto - Canadá \\ mariadelmarpastorbravo@gmail.com \\ Carmen Ballesteros Meseguer. Universidad de Murcia - España \\ carmenbm@um.es
}

Ana Myriam Seva Llor. Universidad de Murcia - España

anamyriam.seva@um.es

Florentina Pina-Roche. Universidad de Murcia - España

florpina@um.es

\section{Resumen}

Objetivo: Conocer las creencias, actitudes y prácticas de los adolescentes en sus relaciones de noviazgo y caracterizar el perfil de los adolescentes que reproducen y que son más susceptibles de sufrir violencia de género.

Método: Diseño observacional, descriptivo, transversal y analítico a través de 3 cuestionarios a 131 hombres y 139 mujeres adolescentes de Educación Secundaria de la Región de Murcia (España).

Resultados: Los adolescentes con padres de bajo nivel educativo y los hombres reconocen en menor medida las formas de violencia y justifican en mayor proporción los comportamientos violentos de control y poder sobre las mujeres. Tanto hombres como mujeres perpetúan la violencia, pero ellos ejercen más la violencia sexual mientras que ellas la verbal, que al mismo tiempo es más sufrida por las adolescentes.

Conclusiones: Las adolescentes son más susceptibles de sufrir violencia verbal y violencia sexual por parte de sus parejas, conformando un grupo de riesgo de violencia de género sobre el que hay que implementar medidas de prevención, detección y protección. Es necesario sensibilizar y formar en igualdad de género, especialmente, a los adolescentes con padres de bajo nivel educativo y los de sexo masculino

Palabras clave violencia de pareja, adolescente, agresión, sexismo, género y salud, relaciones interpersonales, violencia sexual. 


\begin{abstract}
Objective: To determine the beliefs, attitudes and practices of adolescents in their relationships and to characterize the profile of adolescents who reproduce and who are more susceptible to suffer gender violence.

Method: Observational, descriptive, cross-sectional design through self-administered questionnaires to 131 men and 139 women adolescents from secondary education centers in the Region of Murcia (Spain).

Results: Adolescents with parents of low educational level and adolescent men recognize forms of violence to a lesser extent than women and justify to a greater extent, violent behaviors of control and power over adolescent women. Both men and women perpetuate violence, but men exert more sexual violence while women exert more verbal violence. At the same time, adolescent women are most susceptible to verbal violence from their intimate partners.

Conclusions: Female adolescents are more susceptible to verbal violence and sexual violence by their partners, forming a group at risk of gender violence for which prevention, detection and protection measures must be implemented.

It is necessary to sensitize and train in gender equality, especially, to adolescents with parents of low educational level and males.
\end{abstract}

Keywords intimate partner violence, adolescent, aggression, sexism, gender and health, interpersonal relations, sexual violence.

\title{
1. Introducción
}

La violencia en la pareja se define como cualquier comportamiento, dentro de una relación íntima, que cause o pueda causar daño físico, psíquico o sexual a los miembros de la relación (Organización Panamericana de la Salud, 2014). Los adolescentes son especialmente vulnerables a este tipo de violencia ya que constituyen un grupo con una intersección de desventajas sociales y adversidades específicas para contrarrestar sus efectos sobre el bienestar y moldear su propio destino (Rodríguez Vignoli, 2001).

Las actitudes comportamentales violentas en la adolescencia son habituales (Fonagy, 2003). De hecho, los adolescentes ejercen violencia en el aula principalmente como forma de diversión y mecanismo de resolución de problemas (Pina-Roche, F., Seva Llor, AM., Pastor Bravo MM, Ballesteros Meseguer,C., 2016). Según los autores, casi todos los adolescentes, en algún momento, cometen conductas anti-normativas y violentas, pero solamente un $6 \%$ se convierten en agresores o violentos persistentes (Fonagy, 2003). Estas trayectorias de vida "violentas" se constatan a los 12-13 años y se continúan más allá de los 17 y 18 años (Fonagy, 2003).

El Instituto Nacional de Estadística (INE, 2016) afirma respecto a la violencia de pareja entre adolescentes españoles, que uno de cada tres jóvenes considera inevitable o aceptable en algunas circunstancias "controlar los horarios de la pareja", "impedir a la pareja que vea a su familia o amistades", "no permitir que la pareja 
trabaje o estudie" o "decirle las cosas que puede o no puede hacer". Así mismo, el 25\% de las jóvenes asegura que su novio la vigila.

Según las cifras del Instituto Nacional de Estadística (INE), 499 menores de 18 años requirieron medidas cautelares o una orden de protección en 2013 ante situaciones de violencia por el compañero íntimo, cifra que aumenta un 15,4\% en 2014 y alcanza 637 en 2015 (Ministerio de Sanidad Servicios Sociales e Igualdad, 2017b).

La violencia en el noviazgo es un fenómeno de gran magnitud y gravedad (CutterWilson, E., Y Richmond, 2011)(Muñoz-Rivas, Graña, O'Leary, \& González, 2007) (Ortega, R., Ortega-Rivera, J. y Sánchez, 2008) que afecta a la salud física, psicológica, sexual, reproductiva y la salud mental, generando depresión, ansiedad y pensamientos suicidas, además de uso abusivo de alcohol, tabaco y drogas, llegando incluso a la muerte (J. Silverman; A. Raj; L. Mucci; J. Hathaway, 2001). En España, desde 2011 hasta la actualidad, siete adolescentes de entre 16 y 20 años han sido asesinadas por sus parejas sentimentales (Ministerio de Sanidad Servicios Sociales e Igualdad, 2017a).

Según McDonell, J., Ott, J., Mitchell (2010) la violencia en las relaciones de intimidad entre adolescentes constituye un grave problema de salud y derechos humanos en el que las víctimas tienden a aceptar y reproducir la violencia en relaciones futuras. En esta línea Furman, W. y Flanagan (1997) explica que las primeras relaciones amorosas marcan las ideas sobre qué esperar y cómo comportarse en la intimidad en la edad adulta.

La caracterización de la violencia en las relaciones afectivo-sexuales que establecen los adolescentes nos ayudaría a establecer un diagnóstico que puede permitir ampliar o rediseñar las intervenciones en materia de atención y prevención en los casos de violencia de género en esta población.

El objetivo del estudio es conocer las creencias, actitudes y prácticas de los adolescentes en sus relaciones de noviazgo y caracterizar el perfil de los adolescentes que reproducen y que son más susceptibles de sufrir violencia de género.

\section{Método}

Estudio observacional, descriptivo, transversal y analítico que se engloba en un proyecto multicéntrico en el que participan universidades de España, Portugal y Brasil titulado "Violência nas relações de intimidade envolvendo adolescentes à luz de gênero e geração: estudo multicêntrico lusohispano".

El escenario de estudio son tres centros de Educación Secundaria de carácter público dependientes de la Consejería de Educación, Formación y Empleo de la Comunidad Autónoma de la Región de Murcia, elegidos por muestreo no probabilístico de conveniencia. Los criterios de inclusión de la muestra fueron: ser adolescentes de entre 13 y 18 años y estar cursando sus estudios en los centros mencionados. Se pasaron 330 cuestionarios, de los cuales se retornaron 270 
cumplimentados válidamente, conformándose la muestra por 131 hombres $(48,51 \%)$ y 139 mujeres $(51,48 \%)$.

Tras contactar con los directores de los centros educativos, se concertó una reunión informativa donde se explicó a los docentes responsables de las aulas seleccionadas el objeto de la investigación y la forma de cumplimentar el cuestionario. El tiempo empleado para autocompletarlo fue de treinta minutos.

La información se recogió a través de tres cuestionarios. El primero es de elaboración propia e incluye los datos sociodemográficos de los adolescentes (nacionalidad, área rural o urbana, religión, personas con las que conviven y curso académico) y el nivel educativo de los padres. El segundo es el instrumento de conocimientos sobre violencia en las relaciones íntimas (VRI) previamente utilizado en Portugal en estudiantes de secundaria (Rodrigues Dixe, MA.; da Silva Amaro de Oliveira Fabião, 2013) y que se tradujo al castellano por dos traductores independientes que llegaron a acuerdos entre las divergencias encontradas, así mismo el cuestionario fue evaluado por dos expertos en violencia en la adolescencia que añadieron las oportunas modificaciones y se comprobó su adecuada comprensión con un grupo de adolescentes de secundaria que lo cumplimentaron en una primera fase piloto. Éste consta de 47 ítems con posibilidad de respuesta "verdadera" o "falsa", y cuanto más elevada sea la puntuación obtenida, más elevado es el nivel de conocimiento sobre VRI de los adolescentes.

En tercer lugar, se utilizó el cuestionario CADRI validado en su versión española (Fernández Fuertes, Fuertes, Pulido, \& Fernández Fuentes, 2006), el cual detecta la existencia de actos violentos en las relaciones de pareja de los adolescentes y se divide en dos sub-escalas denominadas "violencia cometida" y "violencia sufrida".

El estudio fue aprobado por el Comité de Bioética de la Universidad de Murcia. No de expediente: 1189/2015. Previa recogida de la información (entre los meses de noviembre de 2015 y diciembre de 2016), a todos los participantes se les solicitó el consentimiento informado de los padres o representantes legales. En todo el proceso se contemplaron las normas éticas de la declaración de Helsinki.

Para el análisis estadístico descriptivo de la muestra se han empleado los métodos descriptivos básicos, de modo que, para las variables cualitativas, se ha obtenido el número de casos y porcentaje presentes en cada categoría; y para las variables cuantitativas, los valores mínimo, máximo, media y desviación típica. La comparación entre grupos en las variables cualitativas se ha efectuado mediante la prueba Chicuadrado. La correlación entre variables se estudió mediante el coeficiente de correlación lineal de Pearson. La comparación de las variables dependientes (tipos de violencia sufrida y cometida) se realizó mediante el análisis multivariante de la varianza (MANOVA) bajo los supuestos de esfericidad (contraste de esfericidad de Barlett), homogeneidad de matrices de varianzas-covarianzas (contraste $M$ de Box) entre los grupos y homogeneidad univariante (test de Levene). 
El análisis estadístico se realizó con el programa SPSS 23.0 para Windows. Las diferencias consideradas estadísticamente significativas son aquellas cuya $p<0.05$.

\section{Resultados}

Los sujetos de estudio son 270 alumnos de los cuales un $48,5 \%$ son hombres y un $51,5 \%$ mujeres, con un promedio de edad de 15,3 años (Rango: $13-18$, DT = 1,1). El $74,9 \%$ cursan ESO y el $25,2 \%$ Bachillerato. La mayoría son de nacionalidad española y viven en áreas urbanas, con solo una cuarta parte residiendo en zona rural. En relación a la formación de los padres, la mayoría tienen niveles básicos y medios (37,4\% $32,9 \%)$. Los niveles educativos superiores son más escasos tanto en el padre como en la madre $(14,4 \%-17,8 \%)$. La media de edad de su primera experiencia amorosa es de 13,1 años, y la de su primera actividad sexual 14,7 años.

Con respecto al resultado de conocimientos sobre violencia en las relaciones intimas, se ha obtenido una puntuación global media de 34,6 con una DT de 6,3.

En la variable sexo, se establecen diferencias estadísticamente significativas (tabla 1), siendo la puntuación media obtenida para los hombres 33,2, mientras que las mujeres obtuvieron un mayor porcentaje de respuestas correctas: 35,9 (con una $\chi^{2}$ 3,511; $p=0,01$.

Las chicas consideran que la violencia en el noviazgo se produce porque los novios piensan que tienen derecho a imponerse a ellas $(82,6 \%)$, que el sentimiento de rabia genera violencia $(83,3 \%)$ y que los celos son una de las principales causas de violencia en el noviazgo (84,7\%). Igualmente, manifiestan más que ellos que existen casos de violencia en el noviazgo entre los adolescentes de su edad y que la violencia en la pareja es frecuente, así como que esta violencia no acaba después de casarse.

Por otro lado, el triple de chicos, consideran que la violencia en las relaciones íntimas solo aparece en los estratos sociales bajos y que si su pareja les lleva la contraria tienen derecho a grítale en público. Casi la mitad de ellos afirman que cuando tienes novio/a debemos hacer todo aquello que agrada al otro/a. Más chicos afirmaron que el novio/a solo controla al otro/a porque le gusta mucho y que controlar a su pareja es una manifestación de amor. El doble de chicos que de chicas consideran que ejercer el poder sobre el/la novio/a no es violencia y creen tener derecho a escoger los amigos de su pareja. Así mismo, ellas manifiestan en mayor proporción, el posibilitarmantener a sus amigos. Por último, el $88,4 \%$ de las chicas frente al $74,4 \%$ de chicos consideran que obligar al novio/a iniciar una actividad sexual es una forma de violencia sexual.

Tabla 1. Conocimientos de los adolescentes sobre violencia en las relaciones de intimidad según sexo

\section{Sexo}




\begin{tabular}{|c|c|c|c|c|c|c|}
\hline & Ítem & Respuesta & Chicos & Chicas & $\chi^{2}(1)$ & p-valor \\
\hline $\begin{array}{l}\text { La Violencia en el } \\
\text { noviazgo se produce }\end{array}$ & $P_{2}$ & V & $\begin{array}{r}91 \\
(69,5)\end{array}$ & $114(82,6)$ & 6,40 & $0,011 *$ \\
\hline $\begin{array}{l}\text { porque los novios/as } \\
\text { piensan que tienen } \\
\text { derecho a imponerse a } \\
\text { su pareja. }\end{array}$ & & $F$ & $\begin{array}{r}40 \\
(30,5)\end{array}$ & $24(17,4)$ & & \\
\hline $\begin{array}{l}\text { Los celos son una de } \\
\text { las principales causas }\end{array}$ & $P_{3}$ & V & $\begin{array}{r}87 \\
(66,4)\end{array}$ & $116(84,7)$ & 12,15 & $\begin{array}{r}< \\
0,001 * * *\end{array}$ \\
\hline $\begin{array}{l}\text { de violencia en el } \\
\text { noviazgo. }\end{array}$ & & $F$ & $\begin{array}{r}44 \\
(33,6)\end{array}$ & $21(15,3)$ & & \\
\hline $\begin{array}{lcc}\text { Existen casos } & \text { de } \\
\text { violencia } & \text { en } & \text { el }\end{array}$ & $P_{5}$ & V & $\begin{array}{r}98 \\
(75,4)\end{array}$ & $122(88,4)$ & 7,72 & $0,005^{* *}$ \\
\hline $\begin{array}{l}\text { noviazgo entre } \\
\text { jóvenes de mi edad. }\end{array}$ & & $F$ & $\begin{array}{r}32 \\
(24,6)\end{array}$ & $16(11,6)$ & & \\
\hline $\begin{array}{l}\text { La violencia en el } \\
\text { noviazgo no es }\end{array}$ & P6 & V & $\begin{array}{r}65 \\
(49,6)\end{array}$ & $36(26,3)$ & 15,54 & $\begin{array}{r}< \\
0,001 * * *\end{array}$ \\
\hline frecuente. & & $F$ & $\begin{array}{r}66 \\
(50,4)\end{array}$ & $101(73,7)$ & & \\
\hline $\begin{array}{l}\text { La violencia en el } \\
\text { noviazgo solo aparece }\end{array}$ & $P_{12}$ & V & $\begin{array}{r}22 \\
(16,8)\end{array}$ & $8(5,8)$ & 8,20 & $0,004 * *$ \\
\hline $\begin{array}{l}\text { en los estratos sociales } \\
\text { bajos. }\end{array}$ & & $F$ & $\begin{array}{r}109 \\
(83,2)\end{array}$ & $130(94,2)$ & & \\
\hline $\begin{array}{l}\text { Cuando tienes novio/a } \\
\text { debemos hacer todo }\end{array}$ & $P_{13}$ & V & $\begin{array}{r}64 \\
(49,2)\end{array}$ & $30(21,7)$ & 22,22 & $\begin{array}{r}< \\
0,001 * * *\end{array}$ \\
\hline $\begin{array}{l}\text { aquello que agrada al } \\
\text { otro. }\end{array}$ & & $F$ & $\begin{array}{r}66 \\
(50,8)\end{array}$ & $108(78,3)$ & & \\
\hline $\begin{array}{lr}\text { El novio/a } & \text { solo } \\
\text { controla al otro/a }\end{array}$ & $P_{24}$ & V & $\begin{array}{r}37 \\
(28,2)\end{array}$ & $24(17,4)$ & 4,51 & $0,034^{*}$ \\
\hline $\begin{array}{l}\text { porque le gusta } \\
\text { mucho. }\end{array}$ & & $F$ & $\begin{array}{r}94 \\
(71,8)\end{array}$ & $114(82,6)$ & & \\
\hline $\begin{array}{l}\text { Tenemos derecho a } \\
\text { escoger los amigos de }\end{array}$ & $P_{26}$ & V & $\begin{array}{r}24 \\
(18,3)\end{array}$ & $13(9,5)$ & 4,39 & $0,036 *$ \\
\hline nuestra pareja. & & $\mathrm{F}$ & $\begin{array}{r}107 \\
(81,7)\end{array}$ & $124(90,5)$ & & \\
\hline $\begin{array}{l}\text { Aunque tenga una } \\
\text { relación de pareja } \\
\text { tenqo derecho a }\end{array}$ & $P_{30}$ & V & $\begin{array}{r}117 \\
(89,3)\end{array}$ & $134(97,1)$ & 6,53 & $0,011 *$ \\
\hline $\begin{array}{l}\text { mantener a mis } \\
\text { amigos. }\end{array}$ & & $\mathrm{F}$ & $\begin{array}{r}14 \\
(10,7)\end{array}$ & $4(2,9)$ & & \\
\hline $\begin{array}{l}\text { La violencia no acaba } \\
\text { después de casarse. }\end{array}$ & $P_{32}$ & V & $\begin{array}{r}83 \\
(63,4)\end{array}$ & $105(76,1)$ & 5,17 & $0,023^{*}$ \\
\hline & & $F$ & $\begin{array}{r}48 \\
(36,6)\end{array}$ & $33(23,9)$ & & \\
\hline $\begin{array}{l}\text { Ejercer el poder sobre } \\
\text { el/la novio/a no es }\end{array}$ & $P_{33}$ & V & $\begin{array}{r}32 \\
(24,4)\end{array}$ & $14(10,1)$ & 9,67 & $0,002 * *$ \\
\hline
\end{tabular}


violencia.

Controlar a mi novio/a es una manifestación de amor.

Si mi novio/a me lleva $P_{36}$ la contra tengo derecho a gritarle en público.

El sentimiento de rabia genera violencia.

Obligar al novio/a a $\mathrm{P}_{47}$ iniciar una actividad sexual es una forma de violencia sexual.

$$
\begin{array}{lrrrr}
F & 99 & 124(89,9) & & \\
V & (75,6) & & & \\
V & (18,7) & 8(5,8) & 4,86 & 0,028 * \\
F & 113 & & & \\
V & (86,3) & 130(94,2) & & \\
V & 17(13) & 6(4,3) & 6,40 & 0,011 * \\
& & & & \\
F & 114 & 132(95,7) & &
\end{array}
$$

$V \quad \begin{array}{r}92 \\ (70,8)\end{array} 115(83,3) \quad 6,01 \quad 0,014^{*}$

F $\quad \begin{array}{rr}38 & 23(16,7)\end{array}$

V $\quad 97(74) \quad 122(88,4) \quad 9,16 \quad 0,002 * *$

F $\quad 34(26) \quad 16(11,6)$

Al relacionar el nivel educativo de los padres con los conocimientos sobre VRI, la

\begin{tabular}{|c|c|c|c|}
\hline \multicolumn{3}{|c|}{ Puntuación Grupo } & \multirow[b]{2}{*}{ p-valor } \\
\hline Variable & $\begin{array}{r}\mathbf{I V}, \\
\text { media }(D T)\end{array}$ & Prueba & \\
\hline Sexo & & $t(268)=-3,511$ & 0,001 \\
\hline Hombre & $33,2(6,2)$ & & \\
\hline Mujer & $35,9(6,1)$ & & \\
\hline Curso & & $t(268)=-1,169$ & 0,243 \\
\hline ESO & $34,4(6,2)$ & & \\
\hline $\mathrm{BACH}$ & $35,7(6,7)$ & & \\
\hline Área & & $t(268)=1,692$ & 0,092 \\
\hline Urbana & $35(6,4)$ & & \\
\hline Rural & $33,5(5,8)$ & & \\
\hline $\begin{array}{l}\text { Estudios } \\
\text { padre }\end{array}$ & & $F(2,226)=4,820$ & 0,009 \\
\hline Básico & $33,3(7)$ & & \\
\hline $\mathrm{FP} / \mathrm{BACH}$ & $35,9(5,5)$ & & \\
\hline Superior & $36(5,5)$ & & \\
\hline
\end{tabular}
puntuación global obtenida en el cuestionario de conocimientos es mayor entre aquellos adolescentes cuyos padres tienen estudios superiores (Tabla 2).

Tabla 2. Conocimientos de los adolescentes sobre violencia en las relaciones de intimidad según nivel educativo de los padres 


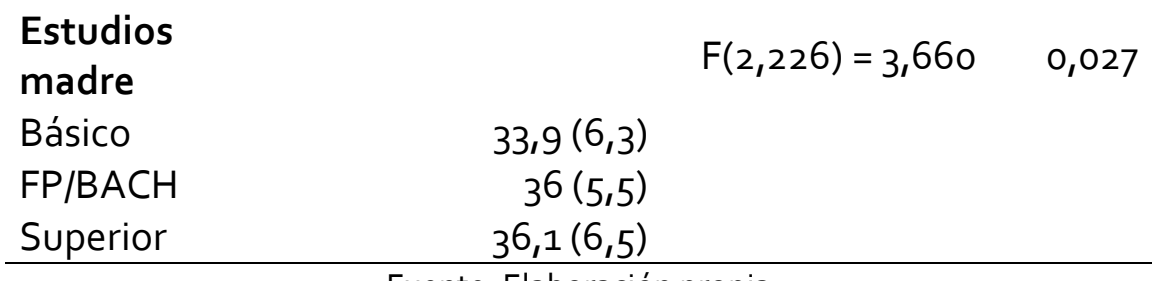

Fuente: Elaboración propia

Se relacionan los estudios básicos del padre con percibir los celos como una señal de amor $\left(\chi^{2=} 6,762 ; p<0,034\right)$ y el control a la pareja como manifestación de amor $\left(\chi^{2}\right.$ $9,204 ; p<0,010)$. Así mismo, se relacionan los niveles de educación básica en ambos padres con la creencia de que el novio/a controla al otro/a porque le gusta mucho (madres: $\chi^{2} 6,05 ; p<0,049-\chi^{2} ;$ padres: $\chi^{2}$ 17,333; $p<0,000$ ), y manifestando que el embarazo no deseado puede ser una consecuencia de violencia en la pareja $\left(\chi^{2} 9,204 ;\right.$ $p<0,010)$. Considerar que el sentimiento de culpa ocurre entre las víctimas de violencia es más frecuente entre los adolescentes cuyas madres tienen estudios básicos $\left(\chi^{2} 8,051 ; p<0,018\right)$. Por otro lado, la creencia de que agradar al otro es un deber en las relaciones de parejas se da con más frecuencia entre adolescentes cuyas madres poseen más estudios $\left(\chi^{2} 7,667 ; p<0,022\right)$.

Con respecto al curso, los estudiantes del nivel de ESO tienen más creencias erróneas respecto a la VRI tales como: la manifestación de que los novios/as provocan violencia por la forma en que se visten $(34,8 \%$ de estudiantes de la ESO frente a un $18,4 \%$ de los de bachillerato con una $\left.\chi^{2} 3,982 ; p<0,046\right)$, que los amigos no comunes perjudican la relación de pareja (23,3\% en los estudiantes de la ESO, frente a un 5,3\% de los de Bachiller con una $\left.\chi^{2} 3,982 ; p<0,046\right)$, y que la violencia en la pareja solo tiene consecuencias psicológicas $(25,2 \%$ de estudiantes de la ESO, frente al 10,5\% de los estudiantes de Bachillerato con una $\left.\chi^{2} 3,958 ; p<0,047\right)$.

Con respecto al área urbana o rural, no hay relación estadísticamente significativa entre diferentes ítems, excepto: la creencia de que la violencia en el noviazgo se produce por derecho de imponerse el uno sobre el otro, más frecuente entre los adolescentes que viven en el área urbana (80\%) frente a los que viven en área rural $(62,6 \%)$ con una $\left.\chi^{2} 5,62 ; p<0,018\right)$. Mas adolescentes del área rural $(47,3 \%)$ frente al $34 \%$ de los que viven en área urbana $\left(\chi^{2} 4,02 ; p<0,045\right)$ consideran poco frecuente la violencia en el noviazgo, así como que ejercer el poder sobre el novio/a no es violencia $(40,5 \%)$ frente al $26,2 \%$ de los que viven en área urbana $\left(\chi^{2} 5,28 ; p<0,022\right)$. Una mayor proporción de los que viven en área urbana $(21,5 \%)$ frente a la rural $(10,8 \%)\left(\chi^{2} 4,08\right.$; $p<0,043)$ consideran falso que obligar al novio a iniciar una relación sexual sea una forma de violencia sexual.

En la tabla 3 se muestra la media, desviación típica y coeficiente de correlación de Pearson $(r)$ de las formas de violencia para agresores y víctimas. Se observa que hay una asociación directa entre acoso y victimización. 
Tabla 3. Medias, desviaciones típicas y coeficiente de correlación tipos de violencia

\begin{tabular}{lrrr}
\hline Violencia & Agresor & Víctima & $r$ \\
\hline Sexual & $0,2(0,36)$ & $0,26(0,4)$ & $0,616 * * *$ \\
Relacional & 0,07 & $0,18(0,46)$ & $0,462 * * *$ \\
Verbal & $(0,25)$ & \\
Amenazas & $0,5(0,48)$ & $0,54(0,5)$ & $0,715 * * *$ \\
Física & 0,13 & $0,16(0,35)$ & $0,608 * * *$ \\
& $(0,29)$ & & \\
& 0,14 & $0,18(0,41)$ & $0,619 * * *$ \\
& \multicolumn{2}{c}{$(0,34)$} & \\
& \multirow{3}{c}{ F**p $<0,001$} &
\end{tabular}

Los efectos significativos en la violencia cometida se presentaron como violencia sexual en los chicos y como violencia verbal en las chicas (Tabla 4). En cuanto a la violencia sufrida, los efectos significativos se presentaron en la violencia verbal que las chicas sufren más.

Tabla 4. Violencia cometida según sexo y Violencia sufrida según sexo

\begin{tabular}{|c|c|c|c|c|}
\hline \multirow{2}{*}{$\begin{array}{l}\text { Violencia } \\
\text { cometida, } \\
\text { Media (DT) }\end{array}$} & \multicolumn{2}{|c|}{ Sexo } & \multicolumn{2}{|l|}{ ANOVA } \\
\hline & $\begin{array}{r}\text { Chicos } \\
(n=99)\end{array}$ & $\begin{array}{l}\text { Chicas } \\
(n=99)\end{array}$ & F(g.l.); p-valor & eta $^{2}$ \\
\hline Sexual & $\begin{array}{r}0,29 \\
(0,44)\end{array}$ & $0,10(0,20)$ & $\begin{array}{r}F(1,196)=14,23, p< \\
0,001 * * *\end{array}$ & 0,068 \\
\hline Relacional & $\begin{array}{r}0,08 \\
(0,30)\end{array}$ & $0,06(0,19)$ & $F(1,196)=0,32, p=0,573$ & 0,002 \\
\hline Verbal & $\begin{array}{r}0,36 \\
(0,38)\end{array}$ & $0,63(0,53)$ & $\begin{array}{r}F(1,196)=16,69, p< \\
0,001 * * *\end{array}$ & 0,078 \\
\hline Amenazas & $\begin{array}{r}0,14 \\
(0,34)\end{array}$ & $0,12(0,25)$ & $F(1,196)=0,23, p=0,632$ & 0,001 \\
\hline Física & $\begin{array}{r}0,15 \\
(0,37) \\
\end{array}$ & $0,13(0,31)$ & $F(1,196)=0,10, p=0,754$ & 0,001 \\
\hline \multirow{2}{*}{$\begin{array}{l}\text { Violencia sufrida, } \\
\text { Media (DT) }\end{array}$} & \multicolumn{2}{|c|}{ Sexo } & \multicolumn{2}{|l|}{ ANOVA } \\
\hline & $\begin{array}{r}\text { Chicos } \\
(n=99)\end{array}$ & $\begin{array}{r}\text { Chicas } \\
(n=99)\end{array}$ & F(g.l.); p-valor & eta $^{2}$ \\
\hline Sexual & $\begin{array}{r}0,27 \\
(0,43)\end{array}$ & $0,25(0,37)$ & $F(1,197)=0,16, p=0,689$ & 0,001 \\
\hline
\end{tabular}




$\begin{array}{lrllll}\text { Relacional } & \begin{array}{r}0,15 \\ (0,46)\end{array} & 0,21(0,46) & F(1,197)=0,80, p=0,371 & 0,004 \\ \text { Verbal } & 0,44 & 0,64(0,54) & F(1,197)=8,41, p=0,004 * * & 0,041 \\ & (0,44) & & & \\ \text { Amenazas } & 0,17(0,37) & 0,16(0,33) & F(1,197)=0,06, p=0,816 & 0,001 \\ \text { Física } & 0,23 & 0,13(0,32) & F(1,197)=3,19, p=0,076 & 0,016 \\ & (0,47) & \end{array}$

Fuente: Elaboración propia

\section{Discusión}

Nuestros resultados muestran que los chicos reconocen en menor medida las formas de violencia y justifican en mayor proporción los comportamientos violentos de control y poder sobre ellas. Esto coincide con diversos estudios, en que ellos presentan un mayor acuerdo con creencias sexistas y justificación de la violencia doméstica (De la Osa Escudero Z, Andres Gomez S, 2013)(Garaigordobil M., Aliri J., 2013), mientras que relacionan el rechazo de la violencia con las chicas jóvenes (McDonell, J., Ott, J., Mitchell, 2010)(De Miguel, 2015). Estas diferencias entre adolescentes dependiendo del sexo pueden haber sido aprendidas a través de los medios de comunicación, los modelos y la familia (Viniegra-Cabello, 2007). De hecho, el $73 \%$ de los adolescentes españoles han escuchado el consejo "los celos son una expresión de amor" de una persona adulta, lo que puede conducir a aceptar dicha afirmación (De Miguel, 2015).

Existe una relación estadísticamente significativa entre el bajo nivel educativo de los padres y considerar los celos y el control como señal de amor, coincidiendo con otro trabajo español que encuentra una mayor justificación de la violencia doméstica a menor nivel socio-económico y cultural de los padres (Garaigordobil M., Aliri J., 2013), y con un estudio Estadounidense que relaciona el nivel educativo de los padres y la victimización en la relación de pareja (Malik, Sorenson, \& Aneshensel, 1997), sin embargo, otras investigaciones defienden que los factores socioeconómicos, nivel educativo y de ingresos de los padres no influyen en la violencia de género en la adolescencia (FOSHEE, 2004)(O'Keefe, 1997).

Atendiendo al curso académico, nuestros resultados indican un menor conocimiento de las consecuencias de la violencia de género, así como un mayor grado de conocimientos erróneos en los estudiantes de ESO frente a los de bachiller, ya que normalizan la dependencia en la pareja al considerar que los amigos no comunes perjudican la relación, así como el control al suponer que la forma de vestirse provoca violencia. Esto se refuerza con estudios que indican que a mayor edad de los estudiantes disminuye el grado de acuerdo con creencias sexistas y la justificación de la violencia doméstica (De la Osa Escudero Z, Andres Gomez S, 2013)(Garaigordobil M., Aliri J., 2013). 
Respecto a la residencia en área rural o urbana, nuestros resultados no muestran una relación estadísticamente significativa en los conocimientos sobre violencia en las relaciones íntimas. Si bien, el alumnado que vive en el área rural identifica, en mayor proporción, el obligar a la pareja a iniciar una relación sexual como una forma de violencia sexual. Esta tendencia se refleja también en un estudio de Vidal (2015) que concluye que el alumnado rural presenta mayor capacidad de reconocer la violencia sexual en la publicidad y es más sensible y contrario a la publicidad sexista que el alumnado urbano.

En relación a la violencia ejercida según el sexo, nuestros datos muestran que los hombres perpetran la violencia sexual mientras que las mujeres ejercen la violencia verbal hacia sus parejas. Otros trabajos reflejan igualmente que la violencia sexual es ejercida en mayor medida por los chicos (Ortega, R., Ortega-Rivera, J. y Sánchez, 2008)(Leen et al., 2013)(Fernández-Fuertes, A.A., Orgaz, B. y Fuertes, 2011)(Gómez, Delgado, \& Gómez, 2014) y que las chicas emplean más habitualmente la violencia psicológica (Muñoz-Rivas et al., 2007) violencia física y verbal emocional (Gómez et al., 2014).

Respecto a la violencia sufrida, se obtuvo un mayor número de mujeres que de hombres como víctimas de violencia verbal emocional. Otras investigaciones que estudian la victimización en las relaciones de pareja coinciden en que las mujeres son más maltratadas y atemorizadas que los hombres (López-Cepero Borrego, RodríguezFranco, Rodríguez-Díaz, Bringas, \& Paíno, 2015), tienden a experimentar más insultos, indiferencia y amenazas por su compañero íntimo (O'Leary, D., Smith, A., Avery-Leaf, S., \& Cascardi, 2008) y que sufren más violencia psicológica (Rivera-Rivera, L., AllenLeigh, B., Rodríguez-Ortega, G., Chávez-Ayala, R. \& Lazcano-Ponce, 2007).

En cuanto a las limitaciones del estudio, son las propias de un diseño transversal. La selección de los tres centros educativos fue por conveniencia, por lo que no podemos asegurar la representatividad de la muestra ni extrapolar los resultados a toda la población de adolescentes.

\section{Conclusiones}

A modo de conclusión, tanto hombres como mujeres perpetúan la violencia, pero en el caso de los hombres ejercen más la violencia sexual mientras que ellas la verbal. Por otro lado, la violencia verbal es más sufrida por las adolescentes.

Los hombres adolescentes reconocen en menor medida las formas de violencia y justifican en mayor proporción los comportamientos violentos de control y poder sobre las mujeres adolescentes. Así mismo el déficit de conocimientos en violencia en las relaciones de intimidad se produce más en los adolescentes de cursos de ESO respecto a los de bachiller y entre aquellos cuyos padres tienen bajos niveles educativos. Ante estos datos, correspondería intensificar la sensibilización y 
conocimientos en materia de igualdad de género especialmente entre adolescentes de sexo masculino, los que cursan ESO y aquellos con padres de bajo nivel educativo.

Por otro lado, las mujeres adolescentes objeto del estudio constituyen un grupo de riesgo ante la violencia de género durante el noviazgo sobre el que hay que implementar medidas de prevención, detección y protección.

\section{Bibliografía}

Cutter-Wilson, E., Y Richmond, T. (2011). Understanding teen dating violence: practical screening and intervention strategies for pediatric and adolescence healthcare providers. Current Opinion in Pediatrics, 23, 379-83.

De la Osa Escudero Z, Andres Gomez S, P. G. P. (2013). Creencias adolescentes sobre la violencia de género. Sexismo en las relaciones entre adolescentes. European Journal of Investigation in Health, Psychology and Education, 3, 265-275. http://doi.org/10.1989/ejihpe.v3i3.49

De Miguel, V. (2015). Percepcion de la violencia de genero en la adolescencia y la juventud.

Fernández-Fuertes, A.A., Orgaz, B. y Fuertes, A. (2011). Características del comportamiento agresivo en las parejas de los adolescentes españoles. Psicología Conductual, 19, 501-522.

Fernández Fuertes, A. A., Fuertes, A., Pulido, R. A., \& Fernández Fuentes, A. A. (2006). Evaluación de la violencia en las relaciones de pareja de los adolescentes. Validación del Conflict ( CADRI ) - versión española 1. International Journal of Clinical and Health Psychology, 6(2), 339-358.

Fonagy, P. (2003). Towards a developmental understanding of violence. The British Journal of Psychiatry, 183(3), 6-36. http://doi.org/10.1192/bjp.183.3.190

FOSHEE, V. (2004). Longitudinal predictors of serious physical and sexual dating violence victimization during adolescence. Preventive Medicine, 39(5), 1007-1016. http://doi.org/10.1016/j.ypmed.2004.04.014

Furman, W. y Flanagan, A. S. (1997). The influence of earlier relationships on marriage: An attachment perspective. In En W. K. Halford y H. J. Markman (Eds.), Clinical handbook of marriage and couples interventions (p. 179-202.). Chichester: Wiley.

Garaigordobil M., Aliri J., M.-V. V. (2013). Justificación de la violencia durante la adolescencia: Diferencias en función de variables sociodemográficas. European Journal of Education and Psychology, 6, 83-93. http://doi.org/10.1989/ejep.v6i2.105

Gómez, M. P., Delgado, A. O., \& Gómez, Á. H. (2014). Violencia en relaciones de pareja de jóvenes y adolescentes. Revista Latinoamericana de Psicología, 46(3), 148-159. http://doi.org/10.1016/S0120-0534(14)70018-4

INE. (2016). Estadística de Violencia Doméstica y Violencia de Género. Año 2015. Retrieved from http://www.ine.es/prensa/np972.pdf

J. Silverman; A. Raj; L. Mucci; J. Hathaway. (2001). Dating violence against adolescent girls and associated substance use, unhealthy weight control, sexual risk behavior, pregnancy, and suicidality. Journal of the American Medical Association, 286, 572-579. Retrieved from doi:10.1001/jama.286.5.572.

Leen, E., Sorbring, E., Mawer, M., Holdsworth, E., Helsing, B., \& Bowen, E. (2013). 
Prevalence, dynamic risk factors and the efficacy of primary interventions for adolescent dating violence: An international review. Aggression and Violent Behavior, 18(1), 159-174. http://doi.org/10.1016/j.avb.2012.11.015

López-Cepero Borrego, J., Rodríguez-Franco, L., Rodríguez-Díaz, F. J., Bringas, C., \& Paíno, S. G. (2015). Percepción de la victimización en el noviazgo de adolescentes y jóvenes españoles. Revista Iberoamericana de Psicología Y Salud, 6(2), 64-71. http://doi.org/10.1016/j.rips.2015.04.001

Malik, S., Sorenson, S. B., \& Aneshensel, C. S. (1997). Community and dating violence among adolescents: Perpetration and victimization. Journal of Adolescent Health, 21(5), 291-302. http://doi.org/10.1016/S1054-139X(97)00143-2

McDonell, J., Ott, J., Mitchell, M. (2010). Predicting dating violence victimization and perpetration among middle and high school students in a rural southern community. Children and Youth Services Review., 32, 1458-1463. Retrieved from doi:10.1016/j.childyouth.2010.07.001

Ministerio de Sanidad Servicios Sociales e Igualdad. (2017a). Fichas de menores víctimas mortales. Retrieved from http://www.violenciagenero.msssi.gob.es/violenciaEnCifras/victimasMortales/fic haMenores/home.htm

Ministerio de Sanidad Servicios Sociales e Igualdad. (2017b). Victimas mortales por violencia de genero. 2016.

Muñoz-Rivas, M. J., Graña, J. L., O’Leary, K. D., \& González, M. P. (2007). Aggression in Adolescent Dating Relationships: Prevalence, Justification, and Health Consequences. Journal of Adolescent Health, 40(4), 298-304. http://doi.org/10.1016/j.jadohealth.2006.11.137

O'Keefe, M. (1997). Predictors of dating violence among high school students. Journal of Interpersonal Violence, 12, 546-568.

O'Leary, D., Smith, A., Avery-Leaf, S., \& Cascardi, M. (2008). Gender Differences in Dating Aggression Among Multiethnic High School Students. Journal of Adolescent Health, 42, 473-479. Retrieved from doi:10.1016/j.jadohealth.2007.09.012

Organización Panamericana de la Salud. (2014). Resumen: respuesta a la violencia de pareja y a la violencia sexual contra las mujeres. Directrices de la OMS para la práctica clínica y las políticas. (O. P. de la Salud, Ed.). Washington, DC.

Ortega, R., Ortega-Rivera, J. y Sánchez, V. (2008). Violencia sexual entre compañeros y violencia en parejas adolescentes. International Journal of Psychology and $\begin{array}{llll}\text { Psychological Therapy, } 8, \quad 63-72 . & \text { Retrieved from }\end{array}$ http://www.redalyc.org/html/56o/56080106/

Pina-Roche, F., Seva Llor, AM., Pastor Bravo MM, B. M. C. (2016). The violence among adolescents as a control in the classroom and in dating. Nure Inv., 13, 1-11.

Rivera-Rivera, L., Allen-Leigh, B., Rodríguez-Ortega, G., Chávez-Ayala, R. \& LazcanoPonce, E. (2007). Prevalence and correlates of adolescent dating violence: baseline study of a cohort of 7960 male and female Mexican public school students. Preventive Medicine, 44(6), 477-484.

Rodrigues Dixe, MA.; da Silva Amaro de Oliveira Fabião, J. (2013). N(amor)o (im)perfeito: avaliação de resultados. In U. de I. em C. da S.- Enfermagem (Ed.), Prevenir a violência no namoro - N(amor)o (im)perfeito - Fazer diferente para fazer a diferença. (pp. 71-97). Coimbra. Retrieved from 
http://rr.esenfc.pt/rr/index.php?module=rr\&target=publicationDetails\&pesquisa= \&id_artigo $=2399 \&$ id_revista $=19$ \&id_edicao $=56$

Rodríguez Vignoli, J. (2001). Vulnerabilidad Y Grupos Vulnerables: Un Marco De Referencia Conceptual Mirando a Los Jóvenes. serie población y desarrollo (CEPAL). Retrieved from http://www.eclac.cl/cgibin/getProd.asp?xml=/publicaciones/xml/9/7889/P7889.xml\&xsl=/celade/tpl/pgf.x sl\&base=/tpl/top-bottom.xslt

Vidal, P. (2015). Sexismo y Publicidad: percepción e influencia en el alumnado adolescente. (Universidad de la Laguna, Ed.). http://doi.org/10.4185/cac9o

Viniegra-Cabello, M. (2007). Actitudes y creencias en torno a la violencia en adolescentes de Secundaria.

\section{Cómo referenciar este artículo/How to reference this article:}

Pastor Bravo, M.M.; Ballesteros Meseguer, C.; Seva Llor, A.M.; Pina Roche, F. (2018). Conocimientos, actitudes y prácticas de adolescentes españoles sobre la violencia de pareja. iQUAL. Revista de Género e Igualdad, 1, 145-158, doi: 10.6018/iQual.301161

Pastor Bravo, M.M.; Ballesteros Meseguer, C.; Seva Llor, A.M.; Pina Roche, F. (2018). Conocimientos, actitudes y prácticas de adolescentes españoles sobre la violencia de pareja. [Knowledge, attitudes and practices of Spanish adolescents on intimate partner violence]. iQUAL. Revista de Género e Igualdad, 1, 145-158, doi: 10.6018/iQual.301161 